\title{
Medidas postoperatorias para la prevención de las infecciones del sitio quirúrgico en ortopedia
}

\author{
Postoperative measures for the prevention of surgical site infections in orthopedics

\begin{abstract}
José Manuel Pérez-Atanasio*
* Médico ortopedista, maestro en Ciencias. Coordinador de Investigación

del Hospital Shriners para Niños, Ciudad de México.

Correspondencia: Dr. José Manuel Pérez-Atanasio. Av. Del Imán No. 257, Pedregal de Santa Úrsula, 09820, Alcaldía Coyoacán, CDMX. Correo electrónico: drmanuelperezata@gmail.com
\end{abstract}

\section{RESUMEN}

Las infecciones del sitio quirúrgico en el periodo postoperatorio es una preocupación constante en el ortopedista, ya que ponen en peligro la vida de millones de pacientes cada año, contribuyen a la propagación de la resistencia a los antibióticos y puede ser la causa de conflictos en la relación médicopaciente. En este artículo presentamos las tres principales recomendaciones de la Organización Mundial de la Salud para su prevención: 1. No prolongar el uso de antibiótico profiláctico después de la cirugía. 2. Usar un apósito estándar en las heridas quirúrgicas. 3. Retirar el drenaje de la herida cuando esté clínicamente indicado.

Palabras clave: Infección del sitio quirúrgico, guía de prevención, Organización Mundial de la Salud.

\section{ABSTRACT}

Surgical site infections in the postoperative period are a constant concern of the orthopedist, endangering the lives of millions of patients each year, contributing to the spread of antibiotic resistance, and may be the cause of conflict in the doctor-patient relationship. In this article, we present the three main recommendations of the World Health Organization for its prevention: 1. Do not prolong the use of prophylactic antibiotics after surgery. 2. Use a standard dressing on surgical wounds. 3. Remove wound drainage when clinically indicated.

Keywords: Surgical site infection, prevention guideline, World Health Organization.

\section{INTRODUCCIÓN}

Las infecciones del sitio quirúrgico preocupan a todos los ortopedistas, ya que ponen en peligro la vida de millones de pacientes cada año, contribuyen a la propagación de la resistencia a los antibióticos y puede ser la causa de conflictos en la relación médico-paciente. La Organización Mundial de la Salud (OMS) realizó una revisión sistemática de la literatura en su Guía para la prevención de la infección del sitio 
quirúrgico, publicada en 2016, ${ }^{1}$ y menciona tres puntos importantes en el periodo postoperatorio para prevenir las infecciones del sitio quirúrgico:

1. Prolongación de la profilaxis con antibiótico.

No se recomienda prolongar el uso de antibiótico profiláctico al término de la cirugía. Este punto tiene un alto grado de recomendación basado en la literatura científica fuerte..$^{2-8}$

Al analizar las guías internacionales para el uso de antibiótico profiláctico en el periodo postoperatorio en pacientes sin factores de riesgo para desarrollar una infección, se identificó que no recomiendan continuarlo después de la cirugía, esto contrasta con las prescripciones habituales que se observan en los hospitales de nuestro país, en los que de manera rutinaria suelen utilizarse antibióticos hasta por siete días después de la cirugía e incluso se continúa por vía oral una vez que el paciente es dado de alta, estas indicaciones las da el médico adscrito y es repetida por los médicos residentes sin analizar la pertinencia de su utilidad.

A continuación, presentamos la Tabla 1 que resume las recomendaciones de las distintas guías con respecto al tiempo que debemos prolongar el uso de los antibióticos profilácticos.

\section{Cobertura de la herida.}

Se sugiere usar un apósito estándar en heridas quirúrgicas cerradas con el fin de prevenir la infección del sitio quirúrgico.

\section{Tabla 1: Recomendaciones del uso de antibióticos profilácticos al término de la cirugía.}

\begin{tabular}{|c|c|}
\hline Guía & $\begin{array}{l}\text { Recomendación del tiempo de uso de los antibióticos profilácticos } \\
\text { después de la cirugía }\end{array}$ \\
\hline SHEA/IDSA (2014) ${ }^{2}$ & Usar hasta por 24 horas después de cualquier procedimiento quirúrgico \\
\hline $\begin{array}{l}\text { American Society of } \\
\text { Health-System Phar- } \\
\text { macists }^{3}\end{array}$ & Suspender dentro de las 24 horas siguientes al término de la cirugía \\
\hline NICE $(2008)^{4}$ & Dar una dosis única del antibiótico profiláctico al iniciar la anestesia \\
\hline $\begin{array}{l}\text { The Royal College of } \\
\text { Physicians of Ireland } \\
(2012)^{5}\end{array}$ & $\begin{array}{l}\text { Usar hasta por } 24 \text { horas después de cirugías ortopédicas. En caso de cirugías a } \\
\text { corazón abierto se puede utilizar hasta por } 48 \text { horas }\end{array}$ \\
\hline $\begin{array}{l}\text { USA, Institute for } \\
\text { Healthcare Improvement: } \\
\text { surgical site infection } \\
(2012)^{6}\end{array}$ & $\begin{array}{l}\text { Suspender dentro de las } 24 \text { horas siguientes al término de la cirugía y en pacientes } \\
\text { con cirugía cardiaca en } 48 \text { horas }\end{array}$ \\
\hline $\begin{array}{l}\text { SIGN: Antibiotic } \\
\text { prophylaxis in surgery } \\
(2014)^{7}\end{array}$ & Uso de dosis única, se puede continuar hasta por 24 horas en artroplastias \\
\hline $\begin{array}{l}\text { UK, High impact inter- } \\
\text { vention bundle }(2011)^{8}\end{array}$ & $\begin{array}{l}\text { Aplicar una dosis } 60 \text { minutos antes de la incisión y repetir la dosis si se presenta } \\
\text { sangrado abundante o si se prolonga el tiempo quirúrgico y/o anestésico }\end{array}$ \\
\hline
\end{tabular}

SHEA = Society for Healthcare Epidemiology of America; IDSA = Infectious Diseases Society of America; NICE = National Institute for Health and Care Excellence; SIGN = Scottish Intercollegiate Guidelines Network. Fuente: OMS. ${ }^{1}$ 
Existen distintos tipos de apósitos que la industria farmacéutica promociona como métodos auxiliares para la cobertura de las heridas, con el objetivo de prevenir la infección del sitio quirúrgico. Estos apósitos por sus características tecnológicas se consideran avanzados, los cuales incluyen materiales con hidrocoloides, plata iónica o metálica o PHMB (Polyhexamethylene biguanide).

Existe la duda razonable de si estos apósitos avanzados son superiores a un apósito estándar, es decir, a la gasa convencional; sin embargo, la OMS y la organización Cochrane, en las revisiones sistemáticas que realizaron, no encontraron alguna diferencia entre el uso de estos dos elementos. ${ }^{1,9,10}$

Estos resultados nos hacen reflexionar sobre el balance costo-beneficio de utilizar apósitos avanzados para el cuidado de las heridas, principalmente en instituciones de salud públicas.

\section{Retiro oportuno del drenaje.}

La OMS sugiere retirar el drenaje de la herida cuando esté clínicamente indicado. No hace una recomendación del momento óptimo para la extracción del drenaje de la herida con el fin de prevenir la infección del sitio quirúrgico.

Cuando colocamos un drenaje al terminar una cirugía en ortopedia, lo cual es una práctica habitual, surge la pregunta: ¿cuándo retirarlo? Para ello no existe un consenso en la respuesta, por lo que la OMS sugiere apegarse a criterios clínicos: cuando el drenaje recolecte $100 \mathrm{ml}$ o menos en 24 horas, o bien, cuando se presente afectación a estructuras funcionales. ${ }^{1}$

\section{CONCLUSIÓN}

Con base en lo discutido anteriormente, podemos concluir con las siguientes recomendaciones para prevenir las infecciones del sitio quirúrgico en el periodo postoperatorio en ortopedia:

1. No prolongar el uso de antibiótico profiláctico después de la cirugía.

2. Usar un apósito estándar en las heridas quirúrgicas.

3. Retirar el drenaje de la herida cuando esté clínicamente indicado.

\section{BIBLIOGRAFÍA}

1. Global Guidelines for the Prevention of Surgical Site Infection. World Health Organization. 2016.

2. Anderson DJ, Podgorny K, Berrios-Torres SI, Bratzler DW, Dellinger EP, Greene L, et al. Strategies to prevent surgical site infections in acute care hospitals: 2014 update. Infect Control Hosp Epidemiol. 2014; 35 (6): 605-627.

3. Bratzler DW, Dellinger EP, Olsen KM, Perl TM, Auwaerter PG, Bolon MK, et al. Clinical practice guidelines for antimicrobial prophylaxis in surgery. Am J Health Syst Pharm. 2013; 70 (3): 195-283.

4. Leaper D, Burman-Roy S, Palanca A, Cullen K, Worster D, Gautam-Aitken E, et al. Prevention and treatment of surgical site infection: summary of NICE guidance. BMJ. 2008; 337.

5. Preventing surgical site infections. Key recommendations for practice. Dublin: Joint Royal College of Surgeons in Ireland/Royal College of Physicians of Ireland Working Group on Prevention of Surgical Site Infection; [Accessed 25 July 2016] 2012, Available in: https://www.rcpi.ie/wpcontent/uploads/2016/01/ Preventing-Surgical-Site Infections-Key-Recommendations-for- Practice.pdf. 
6. Friese S, Willems FT, Loriaux SM, Meewis JM. Prophylaxis in gynaecological surgery: a prospective randomized comparison between single dose prophylaxis with amoxycillin/clavulanate and the combination of cefuroxime and metronidazole. J Antimicrob Chemother. 1989; 24 (Suppl. B): 213-216.

7. Scottish Intercollegiate Guidelines Network. Antibiotic prophylaxis in surgery. [Accessed 10 May 2016] July 2008, updated April 2014. Edinburgh: Healthcare Improvement Scotland. Available in: http://www. sign.ac.uk/pdf/sign104.pdf.

8. High impact intervention; care bundle to prevent surgical site infection. London: Department of Health; [Accessed 24 July 2016] 2011, Available in: http://hcai.dh.gov.uk/files/2011/03/2011-03-14-HII-PreventSurgical-Site-infection- FINAL.pdf.

9. Dumville JC, Walter CJ, Sharp CA, Page T. Dressings for the prevention of surgical site infection. Cochrane Database Syst Rev. 2011; (7): CD003091.

10. Dumville JC, Gray TA, Walter CJ, Sharp CA, Page T. Dressings for the prevention of surgical site infection. Cochrane Database Syst Rev. 2014; 9: CD003091. 